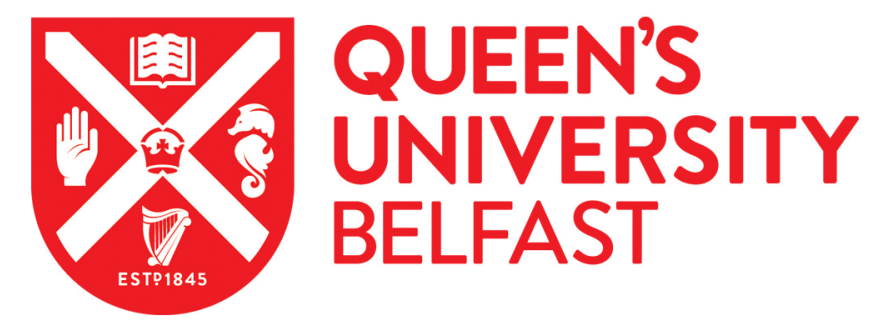

\title{
Significant age-related alterations in the blood plasma metabolome of noncognitively impaired healthy elderly subjects
}

Pan, X., Passmore, P., Graham, S. F., Todd, S., McGuinness, B., \& Green, B. D. (2018). Significant age-related alterations in the blood plasma metabolome of noncognitively impaired healthy elderly subjects. Healthy Aging Research, 7(1), e16. https://doi.org/10.1097/hxr.0000000000000016

Published in:

Healthy Aging Research

Document Version:

Publisher's PDF, also known as Version of record

Queen's University Belfast - Research Portal:

Link to publication record in Queen's University Belfast Research Portal

\begin{abstract}
Publisher rights
Copyright ( 2018 The Authors. Published by Wolters Kluwer Health, Inc. on behalf of

The International Association for the Study of Pain. This is an open-access article

distributed under the terms of the Creative Commons Attribution-Non Commercial-No

Derivatives License 4.0 (CCBY-NC-ND), where it is permissible to download and share

the work provided it is properly cited. The work cannot be changed in any way or used

commercially without permission from the journal
\end{abstract}

\section{General rights}

Copyright for the publications made accessible via the Queen's University Belfast Research Portal is retained by the author(s) and / or other copyright owners and it is a condition of accessing these publications that users recognise and abide by the legal requirements associated with these rights.

Take down policy

The Research Portal is Queen's institutional repository that provides access to Queen's research output. Every effort has been made to ensure that content in the Research Portal does not infringe any person's rights, or applicable UK laws. If you discover content in the Research Portal that you believe breaches copyright or violates any law, please contact openaccess@qub.ac.uk. 


\title{
Significant age-related alterations in the blood plasma metabolome of noncognitively impaired healthy elderly subjects
}

\author{
Xiaobei Pan ${ }^{a}$, Peter Passmore ${ }^{b}$, Stewart F. Graham ${ }^{c}$, Stephen Todd ${ }^{d}$, Bernadette McGuinness ${ }^{b}$, Brian D. Green ${ }^{a, \star}$
}

\begin{abstract}
Background: Age is a major risk factor for most common neurodegenerative diseases. Although an increased research focus on diseases of aging, there is little information regarding the metabolite changes in the aging blood in healthy older adults. Such information could help us to understand neurocognitive changes associated with aging and also further improve the validity and reproducibility of future metabolite biomarkers for neurodegenerative diseases.

Materials and Methods: The purpose of this study was to assess how the metabolomic profiles of noncognitively impaired elderly participants changes with aging. Using a targeted liquid chromatography-mass spectrometry/MS metabolomics approach, this study examined 17 noncognitively impaired elderly participants (TO; $78.10 \pm 6.30 \mathrm{y}$; mini-mental state exam $=29.29 \pm 0.85$ ) and the same 17 subjects were followed-up $\sim 5$ years later $(T 5 ; 83.29 \pm 6.13$; mini-mental state exam $=27.47 \pm 1.62$ ).

Results: The concentrations of 187 plasma metabolites determined were used to build a partial least squares-discriminant model which found that metabolomic profiles taken 5 years (T5) from baseline (TO) were distinctly different $(R 2=0.95 ; \mathrm{Q} 2=0.90)$. When metabolites levels at T5 were compared with T0, 68 of the 73 phosphatidylcholines, 25 of the 40 acylcarnitines, and 2 of the 14 lysophosphatidylcholines were increased, whereas 3 of the 14 lysophosphatidylcholines and 2 of the 14 sphingomyelins were decreased. Moreover, 20 of the 42 amino acids concentrations were significantly different between the 2 time points. Fourteen amino acids were increased at T5, whereas 6 amino acids were decreased.

Conclusions: The plasma metabolome changes with age in elderly, noncognitively impaired subjects, and this could aid in developing valid and sensitive metabolite biomarkers for human disease.
\end{abstract}

Keywords: Metabolomics, Aging, Blood metabolites, Follow-up study

Aging is a natural biological process that is progressive, timedependent, deleterious in nature and is the greatest known risk factor for many diseases, especially neurodegenerative and vascular disease $^{[1]}$. Neurodegenerative diseases represent neurological disorders characterized by the gradual progression and selective loss of anatomically or physiologically related neuronal

Sponsorships or competing interests that may be relevant to content are disclosed at the end of this article.

${ }^{a}$ Asset Technology Centre, Institute for Global Food Security, ${ }^{b}$ Centre for Public Health, School of Medicine, Dentistry and Biomedical Sciences, Queen's University Belfast, Belfast, Northern Ireland, UK, 'B Beaumont Research Institute, Royal Oak, MI and ${ }^{d}$ Altnagelvin Area Hospital, Western Health and Social Care Trust, Glenshane Road, Londonderry, Northern Ireland, UK

${ }^{*}$ Corresponding author. Address: Northern Ireland Technology Centre, Institute for Global Food Security, School of Biological Sciences, Queen's University Belfast, Cloreen Park, Belfast BT9, Northern Ireland, UK. Tel.: + 02890976541 ;

fax: +02890 976513. E-mail address: b.green@qub.ac.uk (B.D. Green).

Copyright @ 2018 The Authors. Published by Wolters Kluwer Health, Inc. on behalf of The International Association for the Study of Pain. This is an open-access article distributed under the terms of the Creative Commons Attribution-Non Commercial-No Derivatives License 4.0 (CCBY-NC-ND), where it is permissible to download and share the work provided it is properly cited. The work cannot be changed in any way or used commercially without permission from the journal.

Healthy Aging Research (2018) 7:e16

Received 13 October 2017; Accepted 7 December 2017

Published online 19 January 2018

Supplemental Digital Content is available for this article. Direct URL citations appear in the printed text and are provided in the HTML and PDF versions of this article on the journal's website, www.healthyagingresearch.com.

http://dx.doi.org/10.1097/HXR.0000000000000016 systems ${ }^{[2]}$. Impairment of memory is often one of the first symptoms of the majority of neurodegenerative disease. Alzheimer disease (AD), Parkinson disease, amyotrophic lateral sclerosis and Huntington disease are common examples of neurodegenerative disease ${ }^{[3]}$. Biomarker discovery for neurodegenerative disease is currently one of the hottest research topics within this field. Biomarkers are defined as characteristics evaluated as indicators of biological or pathogenic processes, or pharmacological response(s) to a therapeutic intervention ${ }^{[4]}$. For neurodegenerative diseases, biomarkers should be able to distinguish underlying the pathology; detect presymptomatic pathologic changes; and/or monitor cognition decline and response to treatment ${ }^{[5]}$. An ideal biomarker is reproducible, stable over time, widely available and reflects directly the relevant disease process. Blood is a commonly used biofluid for biomarker discovery as it contains thousands of hydrophilic and hydrophobic metabolites that could reflect many complex biological processes across the body ${ }^{[6]}$. In addition, collection of blood samples is a minimally invasive procedure as compared with collection of other tissue samples. Although several biomarkers panels for neurodegenerative diseases, like $\mathrm{AD}$, have been reported and showed good sensitivity and selectivity, unfortunately, these biomarkers lack reproducibility for independent patient cohorts. The reason for poor reproducibility may relate to the classical epidemiological issues. Thus, the investigation of aging on the plasma metabolome, particularly in elderly subjects will help to improve the validity and reproducibility of future metabolite biomarkers. 
Several studies have shown that the levels of some blood metabolites can correlate to human phenotypes (eg, age, sex, region, diet, and blood pressure $)^{[7-13]}$. Ishikawa and colleagues evaluated sex-associated and age-associated differences in blood metabolites and many sphingomyelin species were significantly higher in females than in males. They also observed that the ageassociated differences were more prominent in females than in males $^{[8]}$. Furthermore, $\mathrm{Yu}$ and colleagues applied targeted metabolomics techniques to measure 163 metabolites from participants in Germany and UK aged between 32 and 81 years old and discovered that 71 and 34 metabolites were significantly associated with age in women and men, respectively. This led to the identification of 7 metabolites (C0, C10:1, C12:1, C18:1, SM C16:1, SM C18:1, and PC aa C28:1) which increased with age in both cohorts and 1 metabolite, histidine, which decreased ${ }^{[11]}$. Similarly, Lawton and colleagues measured more than 300 metabolites using gas chromatography-mass spectrometry and liquid chromatography-mass spectrometry in 269 individuals. Among these metabolites more than 100 metabolites were associated with age. Changes in protein, energy, and lipid metabolism including fatty acids, carnitine, $\beta$-hydroxybutyrate and cholesterol, as well as oxidative stress markers (eg, oxoproline, hippurate), were observed with increasing age. By contrast, relative concentration of dehydroepiandrosterone-sulfate was the lowest in the oldest age group $^{[12]}$. Menni and colleagues identified a panel of 22 independent metabolites associated with age. The altered metabolites include 9 lipids, 7 amino acids, 2 intermediates in the energy pathway, 2 xenobiotics, 1 carbohydrate, and 1 nucleotide ${ }^{[13]}$.

In the present study, we performed targeted metabolomics analysis to measure the concentrations of 187 plasma metabolites to obtain fundamental information on the age-associated difference in plasma metabolites levels.

\section{Materials and methods}

\section{Ethics statement}

Appropriate research ethical approval at Queen's University Belfast was sought and obtained. Written informed consent was obtained from all participants.

\section{Study design and participants}

Noncognitively impaired elderly participants [mini-mental state exam (MMSE) $\geq 28$ ] were recruited from 3 population groups: patients attending a geriatric day hospital, patients attending the Elderly Rehabilitation Unit for orthogeriatric rehabilitation, and volunteers attending a variety of older people's clubs. On recruitment, all participants were interviewed by a trained physician, experienced in the assessment and diagnosis of $\mathrm{AD}$.
Table 1

Participant demographics and clinical characteristics.

\begin{tabular}{|c|c|c|}
\hline & Initial & Follow-up \\
\hline$\overline{\text { Age }(y)}$ & $78.10 \pm 6.30$ & $83.29 \pm 6.13$ \\
\hline MMSE score & $29.29 \pm 0.85$ & $27.47 \pm 1.62$ \\
\hline Sex F:M & \multicolumn{2}{|c|}{$11: 6$} \\
\hline Education (y) & & \\
\hline
\end{tabular}

F indicates female; M, male; MMSE, mini-mental state exam.

Nonfasting venous blood was collected into $4.5 \mathrm{~mL}$ Vacutainer Plasma Separator Tubes containing $\mathrm{K}_{2}$ EDTA for plasma separation (Becton Dickinson). After $\sim 5$ years $(5.24 \pm 0.20 \mathrm{y})$ those participants with no cognitive complaints (either subjective or objective) and who were also physically and mentally healthy ( $n=17)$ were followed-up and resampled. The mean follow-up MMSE score was $\geq 26$. Although the MMSE performance for participants reduced, it would not indicate cognitive impairment/ dementia and would be within accepted decline in performance over the 5-year interval between testing. All the followed-up participants were diagnosed as cognitively normal on clinical assessment. Samples were stored long-term at $-20^{\circ} \mathrm{C}$ until use. Details of participant characteristics can be found in (Table 1). The particular advantage of our study is the longitudinal design. There were 194 cognitively intact participants recruited at T0. At T5, 93 participants consented to reassessment, 53 were deceased, 38 refused consent to participate again, and 10 were uncontactable. Of the 93 participants who were reassessed at T5, 10 had evidence of cognitive impairment or dementia. From 83 participants with no cognitive impairment at T5, 17 were randomly selected for metabolomic analysis (Fig. 1). Thus, plasma metabolomic profiles for 17 noncognitively impaired elderly participants $(\mathrm{T} 0 ; 78.10 \pm 6.30 \mathrm{y} ; \mathrm{MMSE}=29.29 \pm 0.85)$ were obtained and the same 17 subjects were followed-up and resampled $\sim 5$ years later $(\mathrm{T} 5 ; 83.29 \pm 6.13 \mathrm{y} ; \mathrm{MMSE}=27.47 \pm 1.62)$.

\section{Targeted metabolomics}

A mass spectrometry-based quantitative targeted metabolomics assay was performed using the Biocrates AbsoluteIDQ p180 (BIOCRATES, Life Science AG, Innsbruck, Austria), as previously described ${ }^{[14]}$ which provides simultaneous quantification of 187 metabolites including amino acids, acylcarnitines, phospholipids and sphingolipids, hexose (glucose), and biogenic amines. The samples were processed as per the instructions of the manufacturer included with the kit and analyzed by using a tandem mass spectrometry (MS/MS) method on a triple-quadrupole mass spectrometer (Xevo TQ-MS, Waters Corporation). Briefly, centrifuged human plasma $(10 \mu \mathrm{L})$ was applied to a

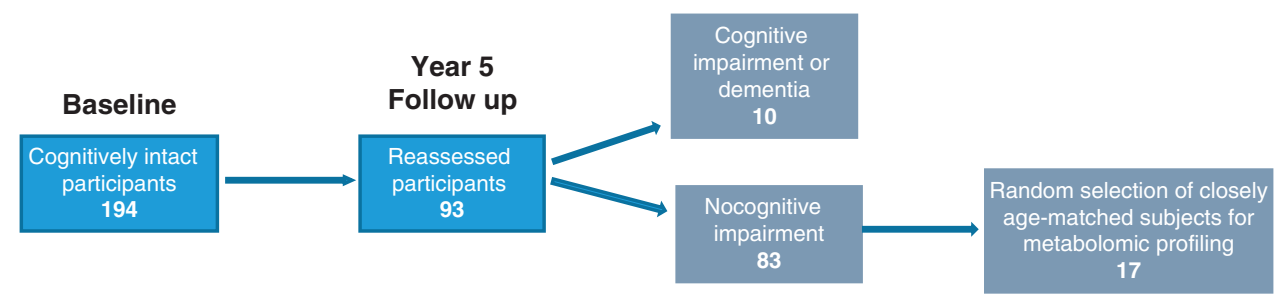

Figure 1. Information of the participant groups. The figure shows the number of participants available for longitudinal metabolomics study. 
Table 2

Instrument parameters for the targeted metabolic assay-UPLC pump settings.

\begin{tabular}{ccccc}
\hline Time & Flow & A\% & B\% & Curve \\
\hline Initial & 0.9 & 100 & 0 & Initial \\
0.25 & 0.9 & 100 & 0 & 6 \\
3.75 & 0.9 & 40 & 60 & 6 \\
3.95 & 0.9 & 40 & 60 & 6 \\
4.25 & 0.9 & 100 & 0 & 10 \\
4.35 & 0.9 & 100 & 0 & 10 \\
\hline
\end{tabular}

Column: Waters ACQUITY UPLC BEH C18 $1.7 \mu \mathrm{m} 2.1 \times 50 \mathrm{~mm}$.

Precolumn: Waters ACQUITY BEH C18 $1.7 \mu \mathrm{m}$ VANGUARD.

96-well filter plate, which contains isotopic internal standards. Amino acids and biogenic amines were derivatized using phenylisothiocyanate and all metabolites were extracted with $5 \mathrm{mmol} / \mathrm{L}$ ammonium acetate in methanol. Amino acids and biogenic amines were analyzed by ultra performance liquid chromatography-mass spectrometry in the multiple reaction monitoring mode. All other metabolites were quantified on the same mass spectrometer but with a flow injection analysis and multiple reaction monitoring methodology. The chromatographic and the mass spectrometry conditions are detailed in Tables 2-4. The concentration of metabolites are expressed as $\mu \mathrm{mol} / \mathrm{L}$.

\section{Statistical analysis}

Metabolite concentrations were exported to Simca 13 (Umetrics, Umea, Sweden) for multivariate analysis. Data were mean centered, pareto scaled, and grouped into initial time point (T0) and 5 years follow-up (T5), and subsequently analyzed using Partial least squares-discriminant analysis (PLS-DA). The performance of the models was evaluated by calculating R2 (cumulative) and Q2 (cumulative). R2 (cumulative) indicates the variation described by all components in the model and Q2 is a measure of how accurately the model can predict class membership. The permutation testing (MetaboAnalyst 3.0 $0^{[15]}$ ) was used to validate the obtained PLS-DA classification models, confirming the results. Univariate analysis of the concentrations for amino acids and acylcarnitines were performed by Wilcoxon matched-pairs signed rank test using Graphpad Prism 5. Heat maps were created using PermutMatrix version 1.9.3.0 $0^{[16]}$.

\section{Results}

\section{Multivariate statistical analysis}

Multivariate statistical analysis was used to provide an initial assessment of plasma metabolite changes between baseline (T0)

Table 3

Instrument parameters for the targeted metabolic assay-flow injection analysis pump settings.

\begin{tabular}{lccc}
\hline Time $(\mathbf{m i n})$ & Flow $(\boldsymbol{\mu L} / \mathbf{m i n})$ & \% A & \% B \\
\hline Initial & 30 & 0 & 100 \\
1.6 & 30 & 0 & 100 \\
2.4 & 200 & 0 & 100 \\
2.8 & 200 & 0 & 100 \\
3.0 & 30 & 0 & 100 \\
\hline
\end{tabular}

\section{Table 4}

Instrument parameters for the targeted metabolic assay-other system settings.

\begin{tabular}{llrr}
\hline & & \multicolumn{2}{c}{ Method } \\
\cline { 3 - 4 } Instrument & \multicolumn{1}{c}{ Parameter } & UPLC & FIA \\
\hline Autosampler & Injection volume & 5 & 10 \\
Column oven & Temperature & 50 & No column \\
MS & Capillary voltage $(\mathrm{kV})$ & 3.2 & 3.9 \\
& Cone voltage $(\mathrm{V})$ & 27 & 22 \\
& Source temp & 150 & 150 \\
& Desolvation temp & 600 & 350 \\
& Cone gas flow $(\mathrm{L} / \mathrm{h})$ & 250 & 0 \\
& Decolvation gas flow $(\mathrm{L} / \mathrm{h})$ & 100 & 650 \\
& Collision gas flow $(\mathrm{mL} / \mathrm{min})$ & 0.15 & 0.15
\end{tabular}

FIA indicates flow injection analysis.

and follow-up (T5). PLS-DA scores plot (Fig. 2) found that metabolomic profiles between baseline and follow-up were easily discriminated by the statistical model $(\mathrm{R} 2=0.97 ; \mathrm{Q} 2=0.90)$. The $P$-value measuring the statistical significance of diagnostic statistics between real and 2000 randomly permuted class labels for the PLSDA model is $<0.0005$. The loadings plots (Fig. 2) of the PLS-DA indicated that the majority of metabolites were significantly different and were widespread from all metabolite classes.

\section{Lipid alterations}

The significant alteration of 101 phospholipids including 14 lysophosphatidylcholine (LysoPCs), 73 phosphatidylcholine (PtdChos), and 14 sphingomyelines (SMs) were quantified for plasma samples collected at both time points (T0 and T5). Three SMs [SM (OH) C24:1, SM C26:0, and SM C26:1] were not measurable in these human plasma samples. Metabolites in the heat map shown in red are upregulated and those in green are downregulated (Fig. 3). There were considerable increases in the levels of PtdChos species, and altered levels of LysoPCs and SMs in the plasma at follow-up. Of the 73 PtdChos measured, only 5 PtdChos were not significantly altered. All of the other 68 PtdChos were significantly increased $(P<0.05)$ in plasma at follow-up. Contrastingly for LysoPCs, the observed alterations in their concentrations were ranged. Two LysoPCs (C14:0 and C28:1) were higher $(P<0.05)$ at follow-up but 3 other LysoPCs (C17:0, C18:0, and C18:1) were significantly lower. Two SMs (C20:2 and C24:1) were significantly lower at follow-up.

\section{Polyamine and arginine metabolism alterations}

The concentrations of 20 amino acids/biogenic amines were significantly different $(P<0.05)$ at follow-up. Putrescine, spermidine, and spermine in the polyamine pathway were all significantly decreased due to aging, even though no disturbance has been found for ornithine. Plasma glutamine levels were found to be elevated while glutamate concentrations decreased significantly. Moreover, asparagine and aspartate concentrations displayed a similar pattern (Fig. 4).

\section{Other metabolites alterations}

Apart from metabolites illustrated in the pathway, many other key metabolites including amino acid, biogenic amines, and 
A

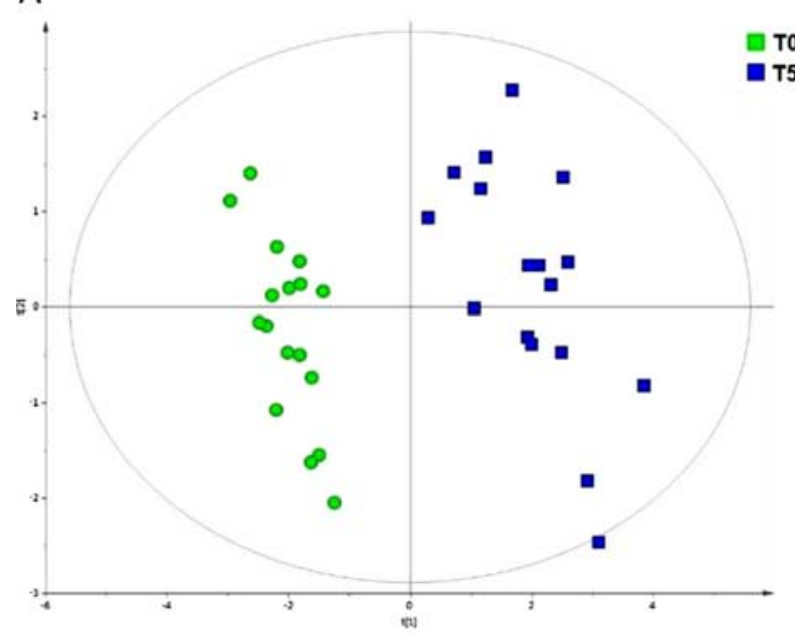

B

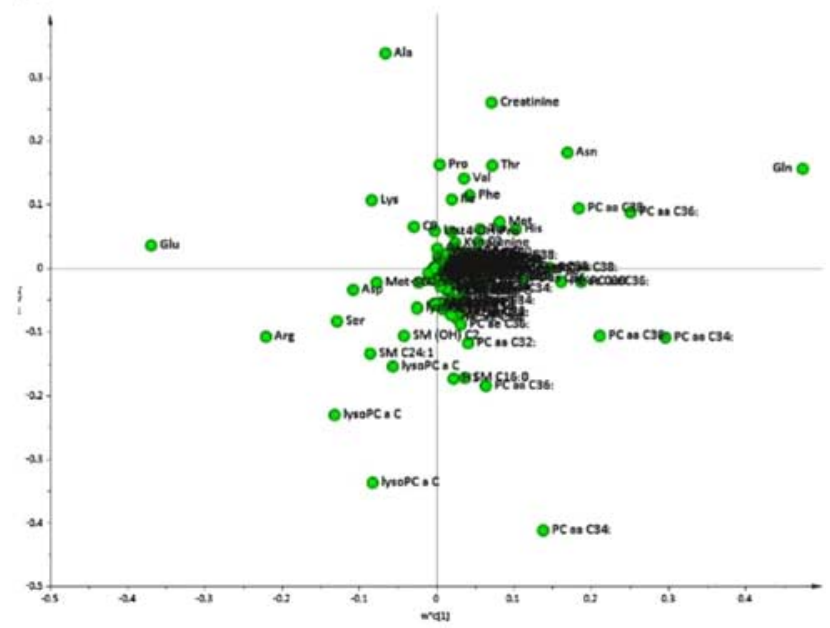

Figure 2. Multivariate statistical analysis clearly differentiates the blood metabolomes of noncognitively impaired subjects at baseline and at 5-year follow-up. A, The scores plot shown the partial least squares-discriminant analysis of 187 blood metabolite concentrations (T0 = green; $\mathrm{T} 5=$ blue). The explained variance (R2) was $97 \%$ and predictive ability (Q2) was 90\%. B, The loading plot shown the majority of metabolites with significant differences.

acylcarnitines were found to be affected due to aging. Histidine, phenylalanine, tyrosine, asymmetric dimethylarginine, kynurenine, symmetric dimethylarginine, and threonine increased significantly at follow-up, while lysine and serine were lower at follow-up (Fig. 5A). Furthermore, there was a general elevation in plasma acylcarnitine moieties (Fig. 5A). Of the 40 acylcarnitines measured, 24 were significantly higher $(P<0.05)$ at follow-up, and only 1 (C12:1) was significantly reduced and the effect was modest $(16.5 \pm 5.7 \%$; $P<0.05$; Fig. 5A). Eleven acylcarnitines were increased by more than $100 \%$ in plasma at follow-up. In order of magnitude these were: $\mathrm{C} 2>\mathrm{C} 3>\mathrm{C} 18>\mathrm{C} 18: 2>\mathrm{C} 4>\mathrm{C} 6(\mathrm{C} 4: 1-$ DC) $>$ C7-DC $>$ C5-DC $($ C6-OH $)>$ C18:1 $>$ C16 $>$ C10.

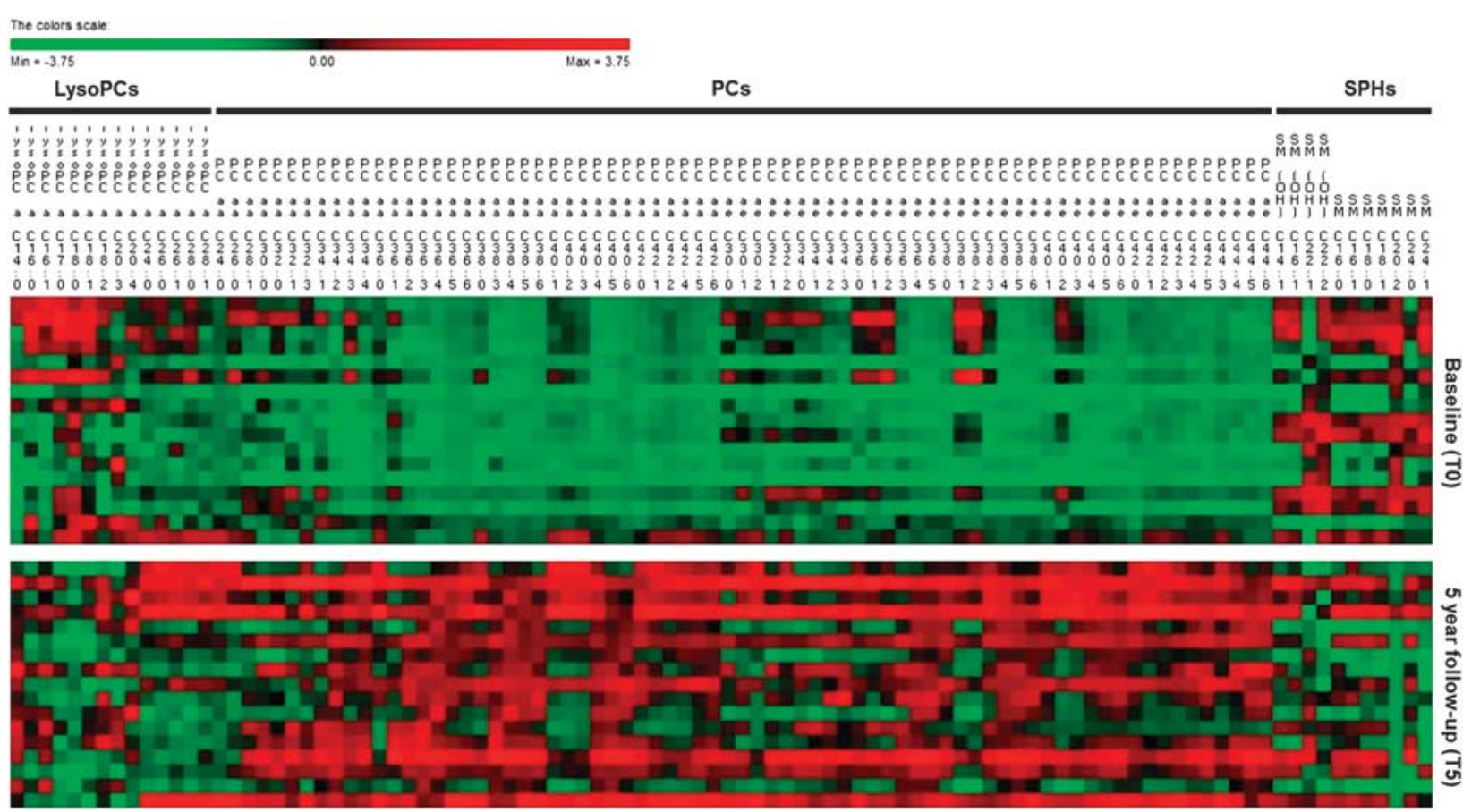

Figure 3. Heat map analysis showing changes in plasma lipids. Each row represents an individual sample and each column an individual lipid moiety. Metabolites shown in red are upregulated and those in green are downregulated. The concentrations of 14 lysophosphatidylcholines (LysoPCs), 76 phosphatidylcholines, and 11 sphingomyelins were significantly different between baseline ( $\mathrm{TO}=$ upper panel) and follow-up ( $\mathrm{T} 5=$ lower panel). Heat map analysis was performed using PermutMatrix software with Z-score normalization and Pearson dissimilarity used as a distance measure. PCs indicates phosphatidylcholines; SPHs, sphingomyelins. 


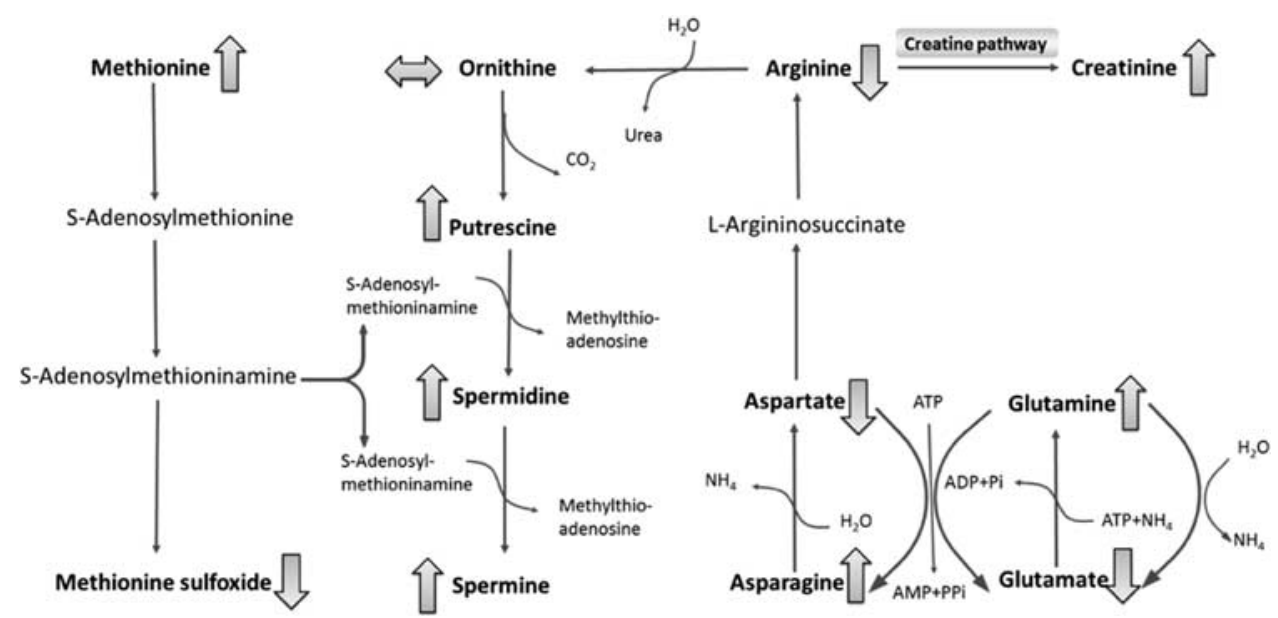

Figure 4. Changes in polyamine, aspartate/asparagine and glutamate/glutamine metabolism. Statistical significance was determined by Wilcoxon matched-pairs signed rank test.

\section{Discussion}

As far as the authors are aware this is the first study to longitudinally investigate metabolic disturbances in human plasma from noncognitively impaired elderly participants using high throughput targeted metabolomics. This study provides important information concerning the validity and reproducibility of potential plasma metabolite biomarkers in ongoing research. First, the time points (T0 and T5) were clearly separated as is evident from the PLS-DA scores plot (Fig. 2) (R2 $=0.97$ and $\mathrm{Q} 2=0.90)$. This indicated that the concentrations of metabolites are strongly associated to advancing age in these specimens. The statistically significant differences were found $<0.0005$ between the values obtained using real and permuted class labels for the PLS-DA models, thus evidencing that the evaluated sample classification was feasible. The loadings plot showed amino acids and phospholipids were the key metabolite classes which were responsible for distinguishing between $\mathrm{T} 0$ and $\mathrm{T} 5$. On the basis of these findings, we examined the changes in the concentrations of the phospholipids across the 2 time points using a heat map analysis (Fig. 3). The heat map clearly illustrates that PtdCho concentrations are significantly elevated at T5. PtdCho and phosphatidylethanolamine are the most abundant glycerophospholipids in the cell membrane. Together with the neutral lipids, they form the characteristic bilayer structure of cellular membranes and regulate membrane integrity. Membrane lipids mainly PtdCho, containing polyunsaturated fatty acids are predominantly susceptible to peroxidation which is a degenerative process that affects unsaturated membrane lipids under conditions of oxidative stress ${ }^{[17]}$. Lipid peroxidation causes some significant modifications to the fatty acids, including the rearrangement or loss of double bonds and, in some cases, the reductive degradation of lipid acyl side chains ${ }^{[18]}$. As a result, lipid peroxidation is believed to contribute significantly to human aging and disease by disrupting the structural conformation, the packing of lipid components and ultimately, the function of biological membranes ${ }^{[19]}$. For the other 2 types of phospholipids measured in this study (LysoPCs and SMs), a small number showed significant disturbances in their concentration between T0 and T5, but without a definite pattern. LysoPCs are the products of partial hydrolysis of PtdChos, where one of the fatty acid groups is removed. SMs are precursors of ceramides. As most of LysoPCs and SMs were not significantly correlated to aging in our study, this suggests that these 2 metabolites classes are potentially better, more stable biomarkers in elderly subjects.

At the 5-year follow-up, we observed significant increases in the 3 main polyamine pathway metabolites (spermine, putrescine, and spermidine). Polyamines are polycations which can interact with negatively charged phosphates in nucleic acids to exert regulatory effects on cellular processes such as cell growth, survival, and proliferation ${ }^{[20]}$. Polyamines are synthesized from ornithine and methionine in many tissues. Although ornithine levels were unchanged, methionine concentrations significantly increased between T0 and T5 specimens. Polyamine levels are affected with aging, but the change may vary considerably among tissues and age groups. Several factors can contribute to this variability; polyamines are absorbed quickly in the intestinal lumen to increase polyamine concentrations in blood and then distributed to almost all organs and tissues in the body, and gut microbiota are also a potential source of polyamines ${ }^{[21,22]}$.

We also found the concentrations of the ornithine precursor arginine to decrease with age. Arginine serves as a precursors of glutamate, urea, proline, creatine, and nitric oxide, which play critical roles in memory formation ${ }^{[23]}$. The increase of nitric oxide (released from a postsynaptic source) leads to an increase in the release of glutamate and, as a result, a stable increase in synaptic transmission, which also linked to memory function ${ }^{[24]}$. Here, we observed the ratio of glutamate to glutamine $(\mathrm{Glu} / \mathrm{Gln})$ and the ratio of aspartate to asparagine (Asp/Asn) both are significantly lower at T5. Glutamine is exclusively synthesized in glial cells mostly from synaptic-born glutamate, and then shuttled back to replenish glutamate neurotransmitter pools in neurons. Thus Gln/ Glu may reflect glutamate-glutamine cycle activity between neurons and glial cells ${ }^{[25]}$, of which the glutamate-glutamine cycle flux was found to be $\sim 30 \%$ lower in healthy elderly humans as compared with younger adults ${ }^{[26]}$. Furthermore, the enzyme L-asparaginase is responsible for catalyzing the deamidation of asparagine and glutamine to aspartate and glutamate, respectively, releasing ammonia in the process ${ }^{[27]}$. A decrease in this enzyme's activity may also result in the decrease of Glu/Gln and Asp/Asn. 

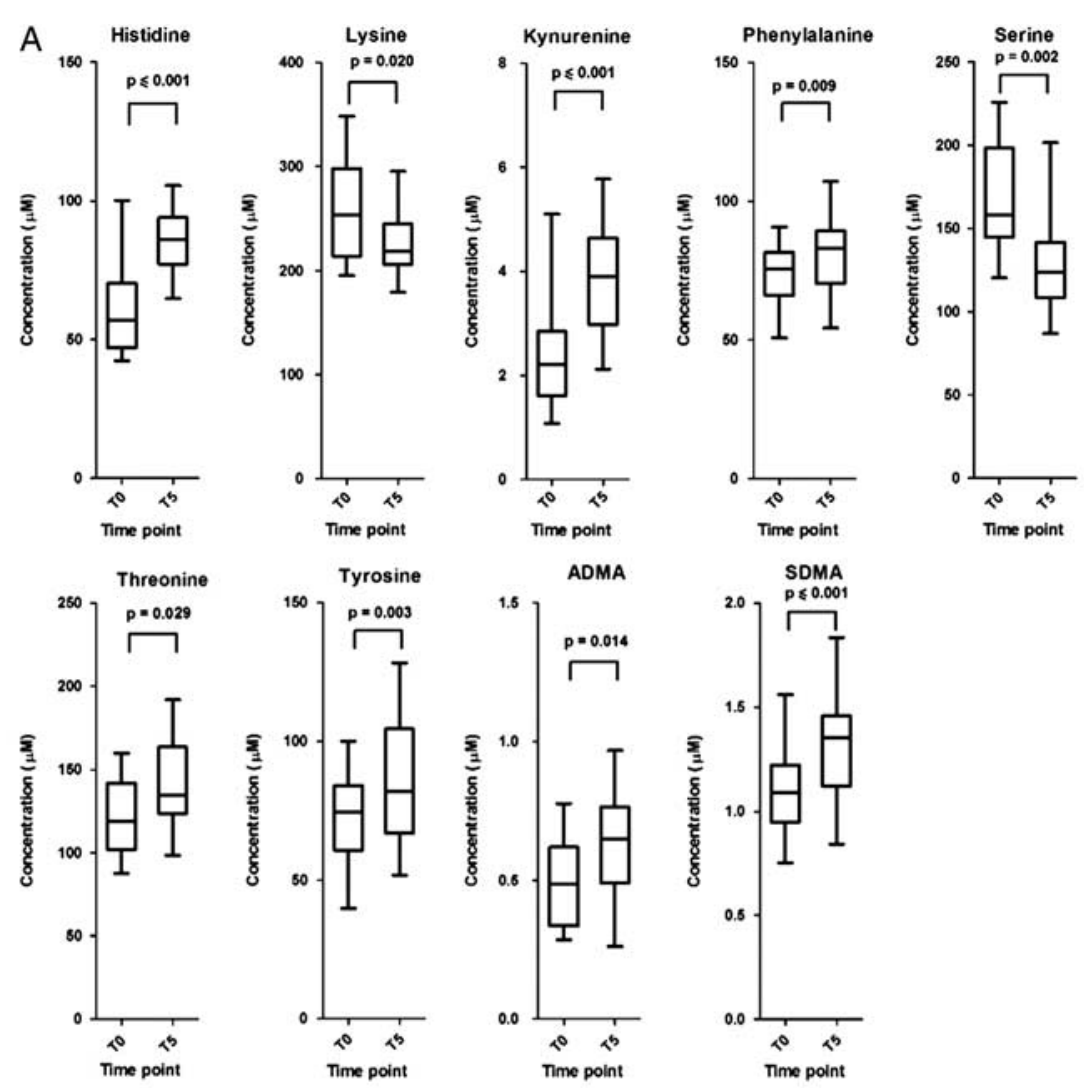

B
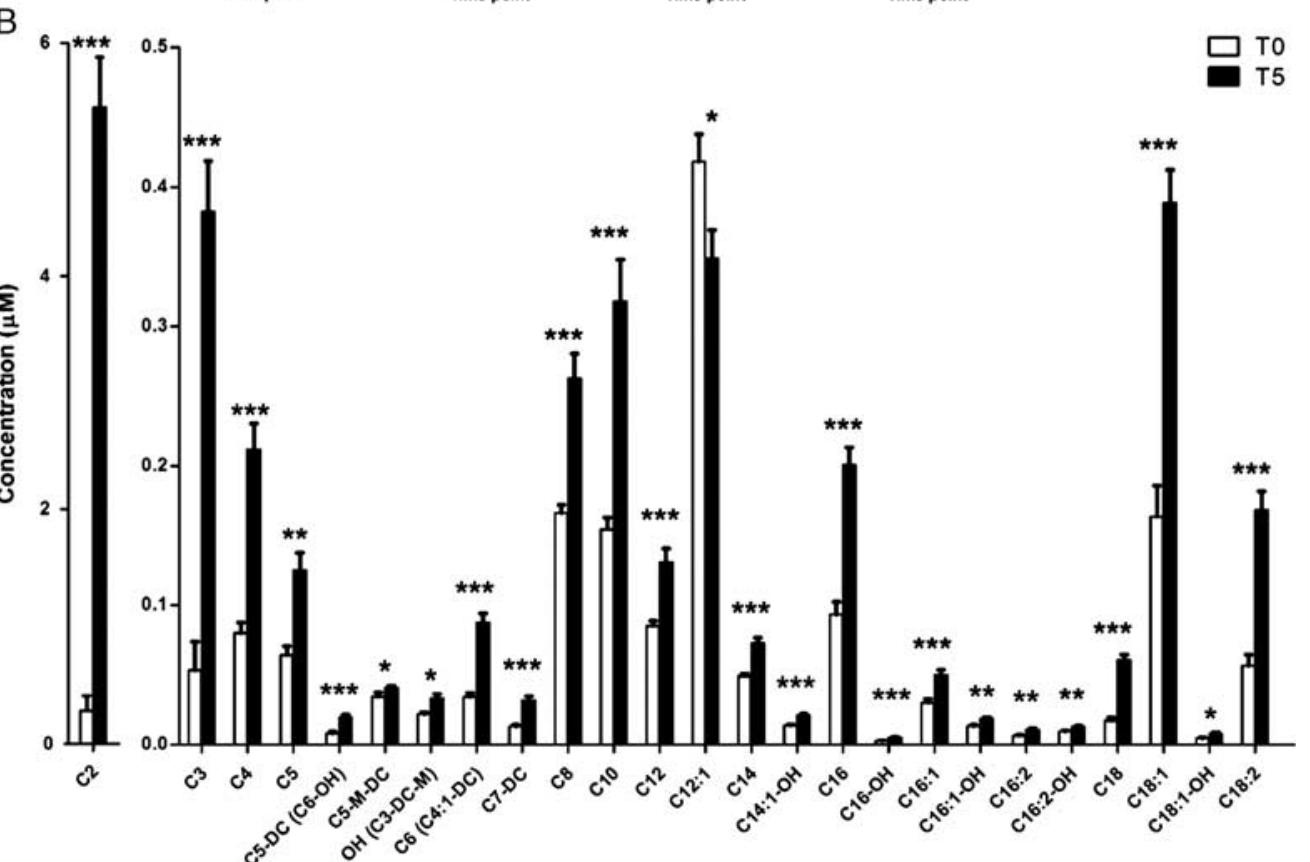

Figure 5. Changes in other amino acids/biogenic amines and acylcarnitines. A, Amino acids/biogenic amines with significant differences between baseline (T0) and follow-up (T5) are shown. The graph shows medians and interquartile ranges. Statistical significance was determined by Wilcoxon matched-pairs signed rank test. $\mathrm{B}$, Changes in plasma acylcarnitine levels with significant differences between baseline (TO) and follow-up (T5) are shown. The majority of acylcarnitine species were increased at follow-up with the exception of $\mathrm{C} 12: 1$ which was decreased. Statistical significance was determined using the Wilcoxon matched-pairs signed rank test $\left({ }^{\star} P \leq 0.05,{ }^{\star \star} P \leq 0.01,{ }^{\star \star \star} P \leq 0.001\right)$. 
Our findings also reveal the alteration of other amino acids/ biogenic amines and acylcarnitines. The primary function of carnitine and acylcarnitine in the cell is as an important transporter of long-chain fatty acids into mitochondria for $\beta$-oxidation, in which carnitine palmitoyltransferase catalyzes the transfer of acyl groups from acyl-Coenzyme A to carnitine to produce acylcarnitines and free coenzyme A. If there is a higher rate of substrate catabolism than energy demand, accumulating acyl-Coenzyme A intermediates are converted back to acylcarnitines, which can then exit cells and tissues ${ }^{[28]}$. As observed from the increase for both membrane phospholipids and acylcarnitines, it could be hypothesized that disturbances to the concentrations of these 2 metabolite classes are attributable to changes in carnitine availability and/or enzyme activity.

Of the significantly different amino acids and biogenic amines due normal aging, many have been suggested to be potential biomarkers or to be involved in the pathophysiological pathway studies for many different diseases, such as cancer, dementia, and diabetes $^{[29-31]}$. We would like to point out 2 general limitations with the current study. First, that the samples provided were nonfasting and which potentially may increase experimental variability among subjects. Second, that the samples were stored long-term at $-20^{\circ} \mathrm{C}$ (rather than $-80^{\circ} \mathrm{C}$ ) which may potentially affect metabolite stability. Nonetheless our results demonstrate the possibility that levels of amino acids, PtdChos, and acylcarnitines are significantly changed by advancing age. In conclusion, these age-associated influences should be taken into consideration in age-matched participant selection and also when selecting blood metabolites as biomarkers in longitudinal studies.

\section{Sources of funding}

Supported by grants from Alzheimer's Research UK (ARUKNCH2012B-5; grant ARUK-PPG2011B-8, ARUK-Network201211 and ARUK-Network2014-16) and a by Proof of Concept grant from Invest Northern Ireland (INI-PoC406).

\section{Author contribution}

X.P.: sample preparation, experimental design, performed experiments, data analysis, wrote manuscript text, reviewed/corrected manuscript. P.P.: clinical and experimental design, reviewed/ corrected manuscript. S.F.G.: experimental design, reviewed/ corrected manuscript. S.T.: collection of clinical samples, clinical assessments of subjects, experimental design, reviewed/corrected manuscript. B.Mc.G.: collection of clinical samples, clinical assessments of subjects, experimental design, reviewed/corrected manuscript. B.D.G.: experimental design, wrote manuscript text, reviewed/corrected manuscript.

\section{Conflict of interest statement}

The authors declare that they have no financial conflict of interest with regard to the content of this report.

\section{References}

[1] Niccoli T, Partridge L. Ageing as a risk factor for disease. Curr Biol 2012;22:R741-52.

[2] Przedborski S, Vila M, Jackson-Lewis V. Neurodegeneration: what is it and where are we? J Clin Invest 2003;111:3-10.
[3] Lin MT, Beal MF. Mitochondrial dysfunction and oxidative stress in neurodegenerative diseases. Nature 2006;443:787-95.

[4] Atkinson AJ, Colburn WA, DeGruttola VG, et al. Biomarkers and surrogate endpoints: Preferred definitions and conceptual framework. Clin Pharmacol Ther 2001;69:89-95.

[5] Ahmed RM, Paterson RW, Warren JD, et al. Biomarkers in dementia: clinical utility and new directions. J Neurol Neurosurg Psychiatry 2014;85:1426-34.

[6] Wishart DS, Jewison T, Guo AC, et al. HMDB 3.0-The Human Metabolome Database in 2013. Nucleic Acids Res 2013;41:D801-7.

[7] Ishikawa M, Tajima Y, Murayama M, et al. Plasma and serum from nonfasting men and women differ in their lipidomic profiles. Biol Pharm Bull 2013;36:682-5.

[8] Ishikawa M, Maekawa K, Saito K, et al. Plasma and serum lipidomics of healthy white adults shows characteristic profiles by subjects' gender and age. PLoS One 2014;9:e91806.

[9] Holmes E, Loo RL, Stamler J, et al. Human metabolic phenotype diversity and its association with diet and blood pressure. Nature 2008;453: 396-400.

[10] Dunn WB, Lin W, Broadhurst D, et al. Molecular phenotyping of a UK population: defining the human serum metabolome. Metabolomics 2015;11:9-26.

[11] Yu Z, Zhai G, Singmann P, et al. Human serum metabolic profiles are age dependent. Aging Cell 2012;11:960-7.

[12] Lawton KA, Berger A, Mitchell M, et al. Analysis of the adult human plasma metabolome. Pharmacogenomics 2008;9:383-97.

[13] Menni C, Kastenmuller G, Petersen AK, et al. Metabolomic markers reveal novel pathways of ageing and early development in human populations. Int J Epidemiol 2013;42:1111-9.

[14] Nkuipou-Kenfack E, Duranton F, Gayrard N, et al. Assessment of metabolomic and proteomic biomarkers in detection and prognosis of progression of renal function in chronic kidney disease. PLoS One 2014;9:e96955.

[15] Xia J, Sinelnikov IV, Han B, et al. MetaboAnalyst 3.0-making metabolomics more meaningful. Nucleic Acids Res 2015;43:W251-57.

[16] Caraux G, Pinloche S. PermutMatrix: a graphical environment to arrange gene expression profiles in optimal linear order. Bioinformatics 2005;21:1280-1.

[17] Girotti AW. Lipid hydroperoxide generation, turnover, and effector action in biological systems. J Lipid Res 1998;39:1529-42.

[18] Reis A, Spickett CM. Chemistry of phospholipid oxidation. Biochim Biophys Acta 2012;1818:2374-87.

[19] Catala A. The ability of melatonin to counteract lipid peroxidation in biological membranes. Curr Mol Med 2007;7:638-49.

[20] Wallace HM, Fraser AV, Hughes A. A perspective of polyamine metabolism. Biochem J 2003;376:1-14.

[21] Pucciarelli S, Moreschini B, Micozzi D, et al. Spermidine and spermine are enriched in whole blood of nona/centenarians. Rejuvenation Res 2012;15:590-5.

[22] Soda K. The mechanisms by which polyamines accelerate tumor spread. J Exp Clin Cancer Res 2011;30:95.

[23] Gad MZ. Anti-aging effects of L-arginine. J Adv Res 2010;1:169-77.

[24] Moncada S, Higgs A. Mechanisms of disease-the L-arginine nitric-oxide pathway. N Engl J Med 1993;329:2002-12.

[25] Duarte JMN, Do KQ, Gruetter R. Longitudinal neurochemical modifications in the aging mouse brain measured in vivo by $\mathrm{H}-1$ magnetic resonance spectroscopy. Neurobiol Aging 2014;35:1660-8.

[26] Boumezbeur F, Mason GF, de Graaf RA, et al. Altered brain mitochondrial metabolism in healthy aging as assessed by in vivo magnetic resonance spectroscopy. J Cereb Blood Flow Metab 2010;30:211-21.

[27] Purwaha P, Lorenzi PL, Silva LP, et al. Targeted metabolomic analysis of amino acid response to L-asparaginase in adherent cells. Metabolomics 2014;10:909-19.

[28] Noland RC, Koves TR, Seiler SE, et al. Carnitine insufficiency caused by aging and overnutrition compromises mitochondrial performance and metabolic control. J Biol Chem 2009;284:22840-52.

[29] Corona G, Polesel J, Fratino L, et al. Metabolomics biomarkers of frailty in elderly breast cancer patients. J Cell Physiol 2014;229:898-902.

[30] Olazaran J, Gil-de-Gomez L, Rodriguez-Martin A, et al. A blood-based, 7-metabolite signature for the early diagnosis of Alzheimer's disease. J Alzheimers Dis 2015;45:1157-73.

[31] Floegel A, Stefan N, Yu Z, et al. Identification of Serum metabolites associated with risk of type 2 diabetes using a targeted metabolomic approach. Diabetes 2013;62:639-48. 